\title{
Combined liver and kidney transplantation in children
}

\author{
Hannu Jalanko • Mikko Pakarinen
}

Received: 7 February 2013 /Revised: 25 March 2013 /Accepted: 8 April 2013 /Published online: 4 May 2013

(C) IPNA 2013

\begin{abstract}
Simultaneous combined liver-kidney transplantation (CLKT) is a rare operation in pediatric patients so that annually only 10-30 operations are performed worldwide. The main indications for CLKT are primary hyperoxaluria type 1 and autosomal recessive polycystic kidney disease. In addition, CLKT is indicated in individual patients with metabolic or cirrhotic liver diseases and end-stage kidney disease. The surgery and immediate post-operative management of CLKT remain challenging in infants and small children. The patients should be operated on before they become severely ill or develop major systemic manifestations of their metabolic disorder. The liver allograft is immunologically protective of the kidney graft in simultaneous CLKT, often resulting in well-preserved kidney function. The long-term outcome after CLKT is nowadays comparable to that of isolated liver and kidney transplantations.
\end{abstract}

Keywords Liver transplantation · Kidney transplantation · Children · Primary hyperoxaluria $\cdot$ Autosomal recessive polycystic kidney disease $\cdot$ Atypical hemolytic uremic syndrome

\section{Introduction}

Combined liver-kidney transplantation (CLKT) is a twostep procedure, in which whole or partial liver and kidney

\section{H. Jalanko $(\triangle)$}

Department of Pediatric Nephrology and Transplantation, Children's Hospital, Helsinki University Central Hospital and University of Helsinki, Stenbackinkatu 11, 00290 Helsinki, Finland

e-mail: Hannu.Jalanko@hus.fi

\section{Pakarinen}

Department of Pediatric Surgery, Children's Hospital,

Helsinki University Central Hospital and University of Helsinki, Helsinki, Finland from a donor are transplanted to a recipient in the same operation. The first successful CLKT was performed at Innsbruck University in 1983, and since then CLKT has become an established treatment modality for both adults and children with severe liver and kidney disorders $[1,2]$. The recent introduction of the MELD (model for end-stage liver disease) scoring system for prioritization of adult liver transplantation, which is strongly influenced by renal dysfunction, has increased the number of CLKT in the USA [3], and currently over 400 CLKT are performed annually in Europe and USA. The great majority of the recipients are adults, and CLKT is still a rare operation in children and adolescents, with only 10-30 pediatric CLKT performed annually worldwide. CLKT accounts for $1-2 \%$ of all pediatric liver transplantations, with one-third of recipients being $0-5$ years of age and two-thirds being between 6 and 17 years old [1].

Combined liver-kidney transplantation is indicated in pediatric patients for three reasons: (1) the patient has a disease leading to both irreversible liver and kidney failure, such as autosomal recessive polycystic kidney disease (ARPKD); (2) the patient has an end-stage kidney disorder (ESRD) caused by a liver-based metabolic disease, such as primary hyperoxaluria type 1 ( $\mathrm{PH} 1)$ or atypical hemolytic uremic syndrome (aHUS), in which case liver is transplanted to correct the underlying defect; (3) the patient has a severe liver disease and poorly functioning kidneys (drug toxicity, vascular complications), in which case concurrent kidney transplantation (KT) is performed to ensure sufficient renal function after liver transplantation (LT). The last indication is common in adults but not in children (Table 1).

\section{Surgical aspects}

Combined liver-kidney transplantation combines the technical aspects of both isolated LT and KT. After hepatectomy, 
Table 1 Indications for pediatric combined liver-kidney transplantation

Major indications

Primary hyperoxaluria type 1 (PH1)

Autosomal recessive polycystic kidney disease (ARPKD)

Minor indications

Liver disease with an occasional kidney failure

-Alpha-1-antitrypsin deficiency

-Glycogen storage disease, type 1

-Alagille syndrome

-Biliary atresia

-Hyperalimentation-induced liver disease

-Primary sclerotic cholangitis

-Liver disorder with a previous kidney transplant (allograft nephropathy)

-Liver tumor with drug nephrotoxicity

Metabolic disease with a controversial experience (see the text)

Atypical hemolytic uremic syndrome (aHUS)

Methylmalonic acidemia (MMA)

the recipient's liver can be replaced by a full size, a reduced size, or a split liver graft (donor liver divided between two recipients) depending on the donor/recipient size match (Fig. 1) [4]. The organs usually come from a deceased adult donor, but living donor CLKT using a left-lateral liver graft and kidney from the same donor (a parent) has also been performed [5]. To ensure enough functional tissue, the weight of the reduced size or split liver should exceed $2 \%$ of the recipient body weight.

The vascular anastomoses are critical in liver graft implanting. Reconstruction of the arterial and portal veins and of liver vein outflow depend on the type of the graft and possible vascular variants of the donor liver. End-to-end anastomoses of the blood vessels are mostly used, but the hepatic artery (from the graft) can also be anastomosed directly to the aorta in small children with narrow vessels. For the biliary reconstruction, end-to-end anastomosis of donor and recipient bile ducts can be performed when a whole liver or a right extended graft is used. A biliodigestive anastomosis with the Roux-en-Y jejunal limb is used with left lateral grafts or when the primary disease affects the biliary tract, such as polycystic diseases and biliary atresia.

After implantation of the liver, a solitary kidney from the same donor is placed extraperitoneally into the right or left iliac fossa, as is the case in isolated KT. When there is a lack of space, the kidney can also be placed into the abdominal cavity. To create enough space for the organs, unilateral or bilateral nephrectomy is often performed in ARPKD patients before CLKT. If organs from a very young donor are used, both kidneys can be transplanted en bloc into the same recipient [6].

Surgical complications after CLKT are not uncommon. Bleeding from the resected liver into the abdominal cavity may require operative revision. Gastrointestinal hemorrhage
Fig. 1 Combined liver-kidney transplantation includes an orthotropic transplantation of a whole, reduced size, or split liver depending on the donor/ recipient size match as well as isolated kidney transplantation from the same donor. The kidney graft is implanted in the right or left iliac fossa or intraperitoneally

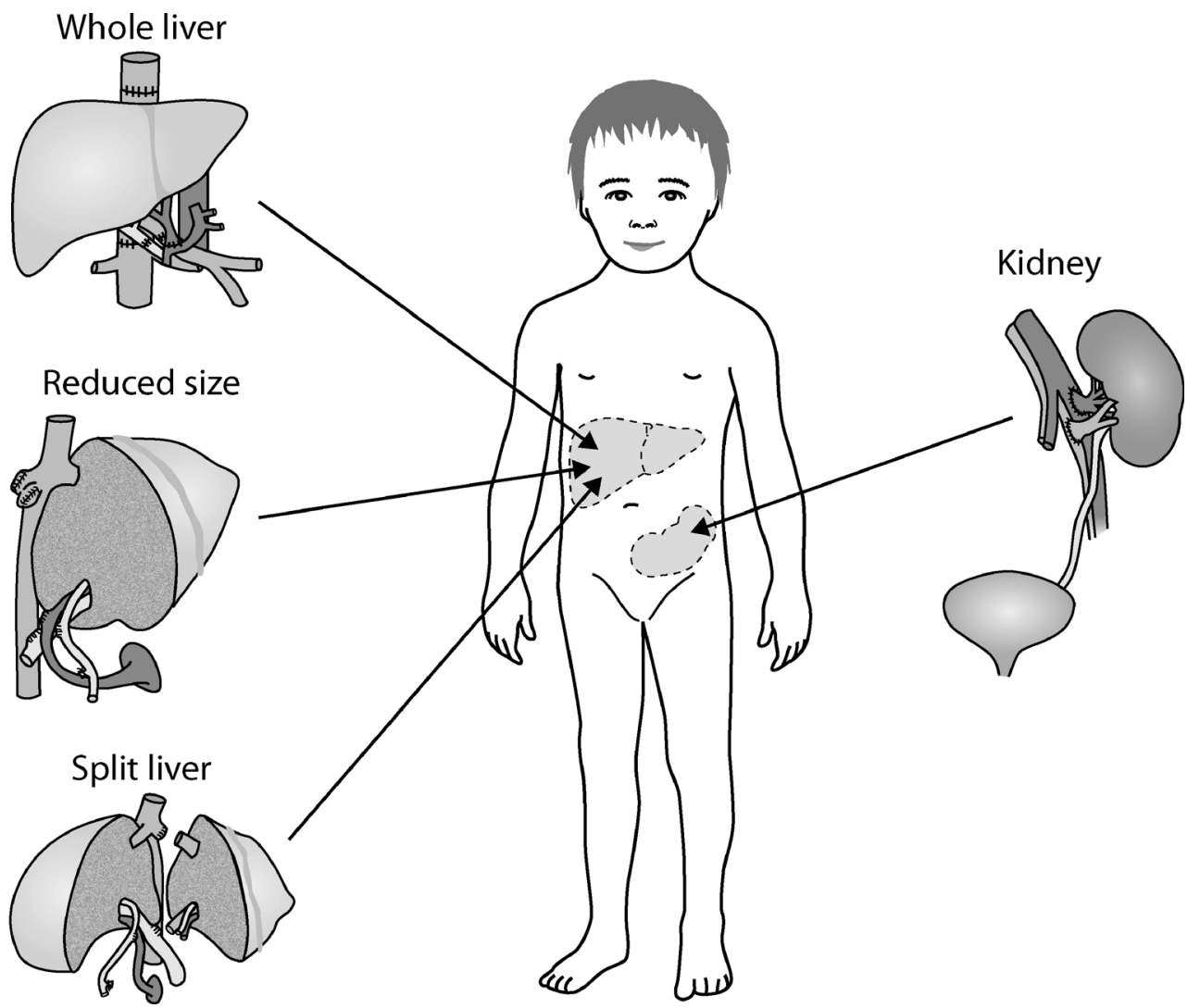


can occur, especially if hemodialysis (requiring anticoagulation) is performed after the operation. Vascular complications include hepatic artery and portal vein thromboses as well as hepatic outflow obstruction. Frequent post-operative Doppler flow measurement (two times a day) during the first few days is important for early detection of circulatory problems. Early thrombectomy or an operative revision of the vascular anastomosis may be needed. A persisting poor blood flow can lead to graft loss and subsequent retransplantation. Also, leak or strictures of the biliary anastomosis may require operative intervention, although a significant proportion of these can be treated with a combination of drainage and stenting. Stents are placed either percutaneously or endoscopically [7]. Little published data are available on surgical problems after CLKT, but after isolated pediatric LT the frequency of bleeding, thrombotic, and biliary complications varies between 5 and $18 \%, 3$ and $20 \%$, and 3 and $35 \%$, respectively $[8,9]$. Surgical complications of the renal graft are less common and include lymphocele and leakage or stenosis of the vesical implantation site or the ureter. Vascular complications may appear, especially if the space for the kidney graft is limited.

After the operation, primary non-function of the liver graft is rare. Delayed function of the kidney graft (DGF) is more common, and patients with PH1 especially have a risk for poor early kidney function, and hemodialysis or continuous hemofiltration may be required. Ventilation is usually needed for 1-2 days only, and the stay in intensive care typically lasts for a few days [10]. Intensive care, however, may last several weeks for patients with multiple problems. As is the case in isolated LT, post-operative ascites formation and intra-abdominal bacterial and yeast infections can require drainage and long-lasting antimicrobial therapy [11].

\section{Immunological aspects}

The immunosuppressive medication of CLKT patients is center-dependent. Induction with anti-interleukin-2-receptor antibodies (basiliximab) or anti-thymocyte globulin (ATG) is used in some, but not all, centers. The use of induction and the slow introduction of calcineurin inhibitor (CNI) have been advocated to reduce post-transplant kidney injury, especially in PH1 patients. Triple immunosuppression with a CNI (cyclosporine A or tacrolimus), antimetabolite (azathioprine or mycophenolate), and glucocorticoid (prednisone or methylprednisolone) is widely used as a maintenance therapy. The immunosuppressive medication of a patient is individualized according to the rejection episodes and drug toxicity. Glucocorticoid is weaned off in some centers.

$\mathrm{ABO}$ blood group compatibility of the donor and recipient is usually required in CLKT. On the other hand, human leukocyte antigen (HLA)-matching and negative T-cell or B-cell cross-match have not been regarded important, as is the case in isolated LT [12]. Retrospective analyses and clinical experience indicate that patients with a positive cross-match do not easily develop hyperacute or acute antibody-mediated rejection (AMR) in either graft [13]. The preformed antibodies are possibly trapped by the liver (Kuppfer cells) without affecting the function of either graft. Thus, the liver offers immunologic protection against AMR of the kidney graft. Recent studies, however, suggest that the liver may not be completely protective [14]. Preformed donor-specific antibodies have been reported to promote AMR, and in a large analysis of over 2,484 CLKT recipients, pre-sensitization had a negative impact on survival of both the patient and the kidney graft [12].

Acute rejection (AR) episodes may appear after the operation in either or both grafts and usually resolve following treatment with high doses of glucocorticoid. Several studies on adult patients; however, have shown that recipients of simultaneous CKLT have a lower incidence of kidney rejection and allograft loss than patients with isolated KT or sequential transplantation [15]. According to the United Network for Organ Sharing (UNOS) database, $21 \%$ of adult CLKT patients had AR episodes as compared to $30 \%$ of patients with isolated KT [16]. The lower rejection rate was more pronounced in sensitized patients and those receiving HLA-mismatched organs. Data on the liver's protective effect are limited in pediatric patients. In a report from the University of California, Los Angeles, there was only one $\mathrm{AR}$ episode in ten CLKT patients, as compared to 16 episodes in $100 \mathrm{KT}$ children [17]. In another pediatric study of 12 patients, a lower AR rate was seen following simultaneous CLKT as compared to sequential transplantation [18]. The lower incidence of rejection is also reflected in the longterm kidney graft function and survival, as recently shown by pediatric and adult data from the UNOS database [17, 19]. In our center the measured long-term glomerular filtration rate (GFR) is on average $23 \%$ better in CLKT patients than in KT children.

The mechanism by which a liver simultaneously transplanted with a kidney would protect the kidney graft from AR episodes, and prolong renal allograft survival, is not known. It has been suggested that CLKT results in microchimerism and donor-specific hyporesponsiveness. Soluble class I HLA-antigens from the liver could neutralize cytotoxic T-lymphocytes and anti-HLA antibodies. Similarly, regulatory HLA-G antigens produced by the liver might inhibit T-cell cytotoxicity [20]. Secretion by the liver of immunomodulatory cytokines has also been suggested. In a recent study of combined auxiliary liver-kidney transplantation, liver grafts strongly up-regulated CCL20 lymphokine, which can recruit dendritic cells and regulatory T-cells and thus prevent rejection [21]. 


\section{CLKT in different indications}

Primary hyperoxaluria type 1

Primary hyperoxaluria type 1 is an autosomal recessive disorder caused by mutations in the $A G X T$ gene encoding vitamin B6-dependent liver-specific peroxisomal enzyme alanine-glyoxalate aminotransferase. This deficiency leads to elevated levels of oxalate and glycolate. Oxalate is excreted in the urine, where it forms crystals with calcium, leading to oxalate stones, nephrocalcinosis, tubular epithelial damage, and renal failure. General oxalosis develops when the GFR declines to $<40-50 \mathrm{ml} / \mathrm{min} / 1.73 \mathrm{~m}^{2}$, leading to oxalate accumulation in bones, skin, blood vessels, myocardium, nerves, joints, and retina, but not in the liver. The mean age of onset of symptoms is 4-6 years, and ESRD is reached at the median age of 24-35 years. Early and aggressive conservative management, including high fluid intake, oxalate crystallization inhibitors, and pyridoxine, may preserve renal function and delay the development of ESRD [22]. Infantile PH1, which nowadays represents almost half of the cases, however, often progresses rapidly, and ESRD is reached before the age of 2-3 years [23]. The most recent recommendations for the diagnostics and management of PH1 were published in 2012 by the OxalEurope Study Group (Table 2) [24].

For over two decades the predominant strategy for management of PH1 in Europe has been to directly pursue CLKT for patients with kidney failure [25, 26]. According to a recent report of the International Primary Hyperoxaluria Registry (IPHR), the use of CLKT as the primary therapy has also increased in non-European centers such that, since 2000, 24 of the 29 transplanted PH1 patients underwent CLKT [27]. CLKT is the method of choice in patients with severe forms of PH1 but not in those who clearly benefit from vitamin B6 therapy and/or have favorable mutations,

Table 2 Suggested transplant strategy in primary hyperoxaluria type 1 according to the stage of the chronic kidney disease of the patient ${ }^{\mathrm{a}}$

1. CKD stage III (GFR 30-60 ml/min/1.73 $\mathrm{m}^{2}$ )

Careful monitoring of the GFR decline

Planning of the transplant strategy

2. CKD stage IV (GFR $15-30 \mathrm{ml} / \mathrm{min} / 1.73 \mathrm{~m}^{2}$ )

Simultaneous liver-kidney transplantation

Sequential liver-kidney transplantation is an option

3. CKD stage V (GFR $\left.<15 \mathrm{ml} / \mathrm{min} / 1.73 \mathrm{~m}^{2}\right)$

Simultaneous or sequential live--kidney transplantation

4. Infantile form

Simultaneous or sequential liver-kidney transplantation

CKD, Chronic kidney disease; GFR, glomerular filtration rate

${ }^{a}$ Modified from the recommendations of the OxalEurope Study Group [24] such as the homozygous c,508G $>$ A mutation [28]. CLKT restores the renal function and corrects the metabolic defect. The new liver, however, does not eliminate the oxalate that has already been deposited in tissues although it does prevent its further overproduction. Oxalate is mobilized from the tissues to plasma and urine so that the oxalate levels remain elevated for up to 3-4 years. Recurrent nephrocalcinosis is still a risk in these patients and may lead to reduced kidney graft function. High fluid intake and crystallization inhibitors are necessary post-transplantation. The aim of the therapy is to reach plasma oxalate levels of $<30 \mu \mathrm{mol} / 1$ and to reduce urinary oxalate excretion $\left(<0.5 \mathrm{mmol} / 1.73 \mathrm{~m}^{2} /\right.$ day $)$ as well as calcium excretion $(0.1 \mathrm{mmol} / \mathrm{kg} /$ day $)$ in order to minimize kidney graft damage. PH1 patients often show a delayed recovery of renal function after CLKT [29], and the use of induction therapy with ATG or basiliximab has been recommended [1], which allows slow and careful introduction of CNIs during the first 1-2 weeks post-transplantation. The benefit of post-transplantation hemodialysis is not clear, but it is often used in patients with significant systemic involvement and high plasma oxalate levels as well as in those with DGF or acute kidney injury [24].

The outcome of PH1 patients after CLKT is quite good. The 5- and 10-year patient survival rates were 80 and $69 \%$, respectively, in a European cohort of 117 PH1 patients transplanted before 2005 [25]. In this cohort, 13 renal grafts failed, of which seven were lost due to oxalate deposition. Better survival following CLKT for PH1 has been attributed to early diagnosis, short time on dialysis, and good condition of the patient (less complications of oxalosis) before the operation. In two recent registry reports, kidney graft survival rates in $\mathrm{PH} 1$ patients were 79 and $84 \%$, respectively, at 3 years [23, 27].

Transplantation strategies for $\mathrm{PH} 1$ have included isolated KT, isolated LT followed by KT (metabolic defect corrected first), or preemptive LT (the patient has stable renal function). Isolated KT, especially using a living donor, has been advocated in solitary patients, such as those who show partial response to pyridoxine therapy. Early reports from the USA suggested equally good outcomes with isolated KT and CLKT [30]. However, more recent data from Europe and USA indicate significantly better outcomes after CLKT [27, 29]. In the European database, only one of $38 \mathrm{PH} 1$ children (3\%) transplanted after 2000 received isolated KT. Successful isolated LT has also been performed in $\mathrm{PH} 1$ patients with a good renal function $\left(\mathrm{GFR}>60 \mathrm{ml} / \mathrm{min} / 1.73 \mathrm{~m}^{2}\right.$ ) [31]. An early LT would theoretically be the best cure, but because of the delay in diagnosis and questionable long-term results the strategy is rarely used.

Atypical hemolytic uremic syndrome

One-third of the patients with aHUS have mutations in genes encoding complement factors $\mathrm{H}$, I, and C3, which 
are synthesized and secreted by the liver. The genetic defects lead to uncontrolled complement activation, which in turn causes kidney capillary damage, deterioration of kidney function, thrombocytopenia, and hemolytic anemia. Isolated KT may correct the renal failure, but due to a very high recurrence rate $(>80 \%)$ it is not a viable treatment modality alone (see below). In contrast to isolated KT, CLKT not only restores kidney function, but also corrects the underlying biochemical defect of the liver.

The early experience on performing CLKT in aHUS patients was disappointing. Severe thromboses appeared perioperatively in the liver, leading to graft loss. Recent studies suggest that this was due to heavy complement activation during the operation [32]. In 2006, a successful CLKT was performed on a 5-year old boy with complement factor $\mathrm{H}$ (CFH) mutations [33]. Before the operation, extensive plasma exchange (PE) was performed to remove the defective $\mathrm{CFH}$. Since then, about 14 aHUS patients have undergone CLKT, of whom 12 are still alive [34]. Guidelines for CLKT in aHUS patients were published in 2009 [35]. These define the patients in whom CLKT is possibly indicated, as well as give instructions for perioperative management, including PE, plasma infusion, and early anticoagulation.

Since the publication of the guidelines for CLKT, interesting data on the use of anti-CF 5 (anti-C5; eculizumab) in aHUS patients with genetic defects in complement components $(\mathrm{CFH}, \mathrm{CFH}, \mathrm{C} 3)$ have been published. This antibody prevents the formation of $\mathrm{C} 5 \mathrm{~b}$ and the $\mathrm{C} 5 \mathrm{~b}-\mathrm{C} 9$ complex (membrane attack complex), and the recent findings indicate that anti-C5 is effective in treating aHUS patients. Moreover, recent data suggest that eculizumab is effective in preventing and treating post-transplant recurrences of aHUS after isolated KT [36]. The first two doses can be given just before and after KT followed by maintenance infusions at 2week intervals. It remains to be seen whether anti-C5 therapy can substitute for CLKT in the management of aHUS. The risk for life-threatening complications is much higher after CLKT than after KT, which strongly speaks for the use of isolated KT and anti-C5 therapy. On the other hand, after a successful CLKT the risk for aHUS recurrence is practically nonexistent, and patients do not need regular and expensive infusions, which further weaken their immunological defenses.

\section{Methylmalonic acidemia}

Methylmalonic acidemia (MMA) is an autosomal recessive disorder caused by complete or partial deficiency of methylmalonyl-CoA-mutase (MCM) or by defects in the synthesis of adenosylcobalamin (cb1A, cb1B). MCM is a mitochondrial enzyme involved in the catabolic pathways of branched amino acids, odd chain fatty acids, and cholesterol. The enzyme defect leads to the accumulation of toxic methylmalonic acid in body fluids and tissues, leading to metabolic acidosis, metabolic stroke, neurological impairment, poor feeding and cardiomyopathy, and pancreatitis, as well as kidney tubular damage and renal failure. The conservative treatment includes a protein-restricted diet, amino acid substitution, L-carnitine, and metronidazole to restrict gut bacterial flora.

MMA is a rare disease $(1: 80,000)$ and to date the criteria for transplantation are not well-established [37]. A literature survey in 2008 identified 27 MMA patients who had undergone transplantation, including six KT, $15 \mathrm{LT}$, and six CLKT [38]. All diagnoses were made within the first 4 months of life, and the average age at transplantation was 9.2 years. Five deaths occurred post-transplantation-four from infection and one due to metabolic decompensation. All patients with CLKT were alive at the time of the literature survey [38]. Common post-operative sequelae included infection, acute rejection, $\mathrm{CNI}$ toxicity, and continued neurologic deterioration. Since this report appeared, only a few cases of MMA with isolated living donor LT or isolated KT have been reported $[39,40]$.

It is clear that none of the transplant modalities are curative for MMA. CLKT or LT decreases the methylmalonic acid levels in urine and plasma but it does not normalize them [37]. Liver and kidney contain only part of the body's MCM activity, and the production of methylmalonic acid from the central nervous system and skeletal muscle continues post-transplant. Neurologic deterioration and metabolic stroke have been documented in several MMA patients post-transplant, and the continuation of protein restriction has been advocated [41]. However, the frequency of metabolic decompensation has decreased significantly in individuals undergoing LT or CLKT despite persistent metabolic abnormalities. LT has been reported to provide a satisfactory quality of life, allowing the mitigation of protein restriction and cessation of tube feeding. Also, LT has been shown to eradicate lethargy and cognitive deficit, which are major symptoms of patients with MMA. It has been proposed that in this clinical context isolated KT might be a safer alternative to CLKT. The transplanted kidney may be sufficient to ensure the partial correction of metabolism and prevent the onset of episodes of metabolic compensation in some patients [40, 42]. At our center, a 10-year old girl with MMA received CLKT 2 years ago; suffered from severe pancreatitis soon after the operation but is now doing clinically well. Her methylmalonic acid levels in plasma and cerebrospinal fluid decreased after CLKT, but remained over 100-fold higher than normal. In general, the genotype of the MMA patient most probably affects the outcome, and since the number of individuals who have undergone CLKT is still small, the detailed effects of transplantation on the underlying metabolic disorder and the overall outcome have yet to be determined. 
Other metabolic diseases

Alpha-1-antitrypsin deficiency ( $\alpha 1$-AT) is the most common genetic disease requiring isolated LT in children. It is an autosomal recessive disorder caused by mutations in the SERPINA1 gene. About $10 \%$ of infants and children with a homozygous Z mutation (Glu342L;PiZZ) develop cholestatic liver disease, and a minority of these progress to cirrhosis and terminal liver failure. Membranoproliferative glomerulonephritis, which in some cases may progress to severe renal failure, is an unusual, associated problem in children and young adults with severe $\alpha 1-\mathrm{AT}$. Reversal of renal dysfunction has been reported in patients with isolated LT. However, CLKT has been a successful procedure in the rare patients with ESRD [43]. Thus, kidney biopsy and evaluation of kidney function have been suggested for all $\alpha 1$-AT patients referred for LT.

Type 1a glycogen storage disease (GSD1a), or von Gierke disease, is a rare, autosomal recessive disease caused by a deficiency of glucose-6-phosphatase, which leads to glycogen accumulation in the liver, kidneys, and intestinal mucosa. Clinical manifestations include hypoglycemia, growth retardation, hepatomegaly, lactic acidemia, hyperlipidemia, and hyperuricemia. Long-term complications include renal disease (mostly focal segmental glomerulosclerosis), osteoporosis, pulmonary hypertension, gout, short stature, and hepatocellular adenomas, all of which may undergo malignant transformation. Isolated LT has been successfully performed in patients with multiple adenomas or with poor metabolic control of the disease, and a small number of GSD1a patients with ESRD have undergone a successful CLKT [44]. Recently, preemptive CLKT has also been performed in a GSD1a patient showing rapid normalization of glucose and lipid metabolism [45]. According to the authors of this study, uremic GSD1a patients with or without adenomas should be considered for early CLKT.

Autosomal recessive polycystic kidney disease

Autosomal recessive polycystic kidney disease is the most common cystic disease of kidney and liver in childhood with an incidence of one in 20,000 live births. ARPKD is caused by mutations in the fibrocystin gene (PKHD1). Fibrocystin is a signaling molecule in kidney tubuli and liver bile ducts, and its genetic defects lead to variable kidney and liver manifestations. In many patients large and non-functioning kidneys require nephrectomy and dialysis in the neonatal period and early kidney transplantation. In others, the kidneys are moderately enlarged, and renal failure develops slowly so that ESRD is reached later in childhood or adolescence $(30 \%$ have ESRD at the age of 10 years). In the ARPKD liver, defective remodeling of the ductal plate, portal fibrosis, and bile duct dilatations cause enlargement of the liver, portal hypertension (gastrointestinal varices, hypersplenism, ascites), and cholangitis episodes. Typically, liver function remains satisfactory for a long time.

Since kidney and liver manifestations progress differently in ARPKD, an individualized management is required. Most often isolated KT is performed first, followed by LT or CLKT later in childhood or adulthood. However, some patients with polycystic disease liver problems proceed quite fast, and LT or CLKT should be regarded as the first option. In a recent literature survey of 116 transplanted ARPKD patients, $56 \%$ had undergone isolated KT, $34 \%$ had LT, and $9 \%$ had CLKT [46].

The decision of the right type of transplantation may be challenging. Although the patients have large liver and portal hypertension, the liver function often remains satisfactory for a long time, and the indications for LT or CLKT are not clear. When isolated KT is performed, hepatic disease can worsen the outcome, especially by increasing the risk for cholangitis and sepsis $[47,48]$. In a retrospective analysis of 203 patients with polycystic kidney disease and isolated $\mathrm{KT}$, nine patients $(4.4 \%)$ died from sepsis posttransplant, which is a high frequency among KT patients. Also, after an isolated KT the quality of life of children with ARPKD often remains impaired due to the large size of the liver and spleen as well as the infectious and bleeding complications. Placement of a portosystemic shunt may help but carries a risk for hepatic encephalopathy, especially in uremic patients. Also, the shunt may need revision because of thrombosis or growth of the child [46, 47, 49].

No general guidelines for the use of CLKT in ARPKD patients have been published. The morbidity and mortality after CLKT are still higher than in isolated KT, which favors the use of isolated KT as the first option. On the other hand, refractory complications of portal hypertension and recurrent cholangitis suggest CLKT as the first option (Table 3). Severe pathological findings of the liver (cirrhosis, large and infected biliary dilatations) and genotype (truncating mutations) also affect the treatment strategy [50]. Many of the children need KT later on, and simultaneous CLKT from the same donor probably provides better long-term kidney function than a sequential transplantation.

\section{Patients with liver failure and kidney dysfunction}

A child with a severe liver disease may occasionally have renal failure not caused by the primary disease. The concomitant dysfunction may be the result of renal vascular thrombosis, urinary tract malformation, glomerulonephritis, or drug toxicity (CNIs used in a previous transplantation; cytostatic drugs used because of liver tumors). In these patients the decision of isolated LT versus CLKT may be 
Table 3 Indications for combined liver and kidney transplantation or isolated liver transplantation in patients with autosomal recessive polycystic kidney disease

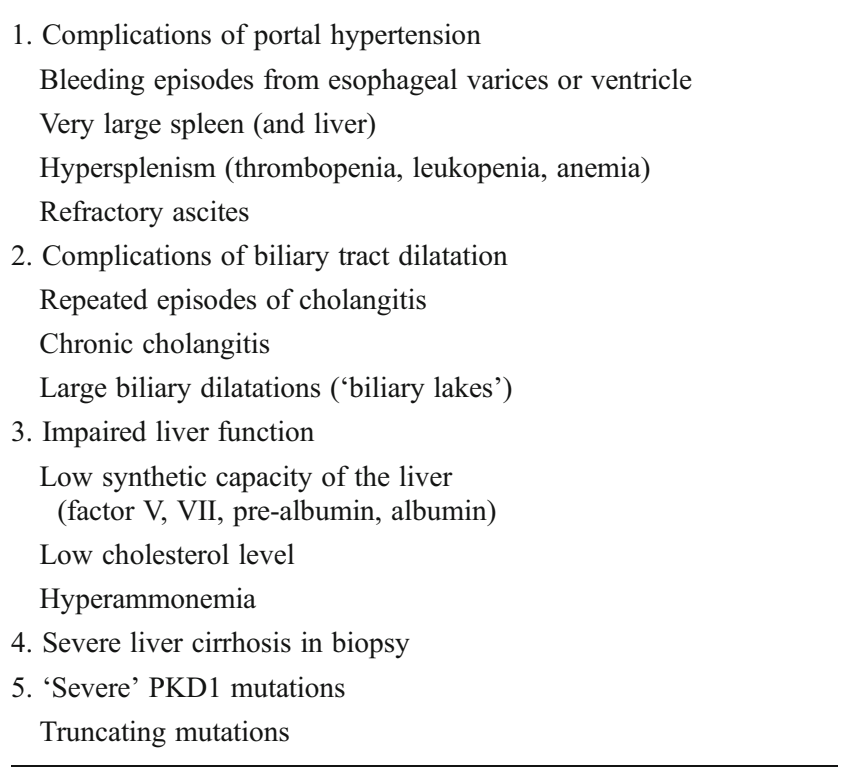

PKD, Polycystic kidney disease

difficult, as the prognosis of kidney function is not clear-cut. While no general recommendations for pediatric patients have been published, the indications in adult patients were addressed by a consensus conference in 2006 [51]. According to these guidelines patients with advanced kidney disease [chronic kidney disease (CKD) stages IV/V; GFR $<30 \mathrm{ml} / \mathrm{min} / 1.76 \mathrm{~m}^{2}$ ] or significant glomerulosclerosis $(>35 \%)$ or with tubulointerstitial fibrosis $(>30 \%)$ on renal biopsy are better served with CLKT. With this approach one can avoid the complications of renal failure in the postoperative period as well as the need for dialysis and KT after LT. This has been clearly demonstrated in adult patients, but data on children are scarce [52].

Patients who develop hepatorenal syndrome (HRS) due to the failing liver, however, often show recovery of the renal function and need only a liver graft. In adults, only a small percentage of LT patients with short-lasting HRS subsequently develop ERSD [51]. On the other hand, those adult patients who need dialysis for over 6 weeks prior to transplantation have a significant risk for persistent renal failure and should be considered for CLKT.

Since the regenerative capacity of the kidneys in children is not very different from that in adults, the published recommendations for adult patients with HRS or other renal problems seem quite valid also for children. However, the strategy must be chosen individually. In cases with structural problems and severe fibrosis in kidney biopsy, the decision is easier than in the rare children with functional problems such as HRS.

\section{Long-term outcome}

The outcome of CLKT is dependent on the primary cause of hepatic and kidney dysfunction as well on as the clinical condition of the patient. While children with a metabolic disease and an early transplantation usually have a good prognosis, patients with multi-organ failure are likely to experience poorer outcome. In adults and adolescents, liver and kidney allograft survival following CLKT is comparable with that seen following isolated LT and KT, respectively. On the other hand, implantation of a reduced size or a split liver with an adult kidney into an infant still presents a technical challenge. In the long term, liver function is usually very stable without evidence of chronic rejection (vanishing bile duct syndrome). However, a slow decrease of kidney function caused by chronic allograft nephropathy is often unavoidable.

Due to the small number of operations in children the outcome data for CLKT are still sparse. Analysis of the 1995-2005 UNOS database revealed that 20.1\% of the 111 pediatric patients with CLKT lost their kidney graft within the first 6 months after transplantation as compared to $5.9 \%$ of patients receiving only kidney graft. However, death-censored 5-year kidney graft survival ( $>6$ months) was significantly better in patients with CLKT as compared to the KT alone group (88 vs. $72 \%$ ) [17]. Outcome data for CLKT in patients with ARPKD are limited. Among 78 patients in the USA, the 1-, 3-, and 5-year survival rates were $79.5,75.5$, and $75.5 \%$, respectively [1]. Similar percentages have been reported in single-center reports [50]. In our center, four of the 14 transplanted ARPKD patients had CLKT as the first operation, and five first underwent $\mathrm{KT}$ followed by CLKT at a later date. Seven of the nine patients are alive (follow-up of 2-14 years), and their quality of life is good.

Transplantation outcome in $\mathrm{PH} 1$ patients has improved over the past decades. Of the $26 \mathrm{PH} 1$ patients in the IPHR, $67 \%$ were alive 7 years after CLKT. Among patients who were transplanted since $2000,84 \%$ of the 29 first kidney grafts (24 CLKT) were functioning in 2010 [27]. In the large European registry, the kidney graft survival rate in $\mathrm{PH} 1$ patients was 82,79 , and $76 \%$ at 1,3 , and 5 years, respectively, for CLKT. These percentages are still about $10 \%$ lower than those observed in patients with isolated KT for reasons other than PH1 [23]. Due to the oxalate load, the recovery of renal function is often delayed in $\mathrm{PH} 1$ patients [29]. Aggressive pre- and post-operative medical management is crucial in $\mathrm{PH} 1$.

\section{Conclusions}

Combined liver-kidney transplantation has become an accepted treatment modality for patients with severe liver and 
kidney disorder. While CLKT is still technically challenging in infants and small children, it is the only curative treatment for several types of congenital disorders, especially PH1 and ARPKD. The early mortality after CLKT is mostly associated with infections and surgical complications of the liver graft. On the other hand, chronic problems with liver graft are quite rare, and the liver most probably protects the kidney allograft from rejection, which results in improved late kidney function and survival.

\section{Key summary points}

The major indications for combined liver-kidney transplantation (CLKT) in children are primary hyperoxaluria (PH1) and autosomal recessive polycystic kidney disease (ARPKD).

Patients should be listed for CLKT before the development of major systemic manifestations of the original disease.

The outcome of CLKT has improved during recent years and is comparable to that of isolated liver or kidney transplantation.

\section{Multiple choice questions (answers are provided following the reference list)}

1. The main indications for combined liver-kidney transplantation (CLKT) in children are:

a. Primary hyperoxaluria type 1 (PH1) and autosomal recessive polycystic kidney disease (ARPKD)

b. PH1 and atypical hemolytic uremic syndrome (aHUS)

c. aHUS and methylmalonic academia (MMA)

d. aHUS and PH1

2. The major life-threatening surgical complication after CLKT is:
a. Ureteral stricture of the kidney graft
b. Thrombosis of liver artery
c. Thrombosis of the kidney artery
d. Biliary stricture

3. The major immunological problem after CLKT is:

a. Acute antibody-mediated rejection of the liver graft

b. Chronic rejection of the liver graft (vanishing bile duct syndrome)

c. Chronic rejection of the kidney graft

d. Acute cellular rejection of the liver

4. CLKT is performed:

a. More often in children than in adults

b. Often in patients with liver failure and hepatorenal syndrome (HRS)

c. More often than isolated kidney transplantation in PH1 patients d. Usually as the first transplantation in ARPKD patients

5. The outcome of CLKT is:
a. Better in infants than in older children and adolescents
b. Better in ARPKD as compared to PH1
c. Better in MMA than in PH1
d. Better in MMA than in ARPKD

\section{References}

1. Sutherland SM, Alexander R, Sarwal MM, Berquist WE, Concepcion W (2008) Combined liver-kidney transplantation in children: Indications and outcome. Pediatr Transplant 12:835-846

2. Chava SP, Singh B, Pal S, Dhawan A, Heaton ND (2009) Indications for combined liver and kidney transplantation in children. Pediatr Transplant 13:661-669

3. Mehrabi A, Fonouni MA, Rahbari AE, Muller SA, Morath C, Seckinger J, Sadeghi M, Golriz M, Esmaieilzadeh M, Hillebrand N, Weitz J, Zeier M, Buchler MW, Schmidt J, Schmied BM (2009) A single center experience of combined liver kidney transplantation. Clin Transplant Suppl 21:102-114

4. Herden U, Kemper M, Granschow R, Klaassen I, Grabhorn E, Brinkert F, Nashan B, Fischer L (2011) Surgical aspects and outcome of combined liver and kidney transplantation in children. Transplant Int 24:805-811

5. Sakamoto S, Kasahara M, Fukuda A, Tanaka H, Kakiuchi T, Karaki C, Kanazawa H, Kamei K, Ito S, Nakazawa A (2012) Pediatric liver-kidney transplantation for hepatorenal fibrocystic disease from a living donor. Pediatr Transplant 16:99-102

6. Heffron TG, Rodriguez J, Fasola CG, Casper K, Pillen T, Smallwood G, Warshaw B, Romero R, Larsen C (2009) Successful outcome after early combined liver and en blockidney transplant in an infant with primary hyperoxaluria type 1 . A case report. Pediatr Transplant 13:940-942

7. Haapamaki C, Udd M, Halttunen J, Lindstrom O, Makisalo H, Kylanpaa L (2012) Endoscopic treatment of anastomotic biliary complications after liver transplantation using romovable, covered, self-expandable metallic stents. Scand J Gastroenterol 47:116-121

8. Sundaram S, Alonso E, Anand R, Study of Pediatric Liver Transplant Research Group (2008) Outcomes after liver transplantation in young infants. JPGN 47:486-492

9. Englesbe MJ, Kelly B, Goss J, Fecteau A, Mitchell J, Andrews W, Krapohl G, Magee JC, Mazariegos G, Horslen S, Bucuvalas J (2012) Reducing pediatric liver transplant complications: a potential roadmap for transplant quality improvement initiatives within North America. Am J Transplant 12:2301-2306

10. Harps E, Brinkert F, Ganschow R, Briem-Richter A, van Husen M, Schmidtke S, Herden U, Nashan B, Fischer L, Kemper MJ (2011) Immediate postoperative intensive care treatment of pediatric combined liver-kidney transplantation: outcome and prognostic factors. Transplantation 91:1127-1131

11. Perera MT, Silva MA, Sharif K, McKiernan PJ, Kelly DA, Lloyd C, Milford DV, Mayer DA, Mirza DF (2009) Improved outcomes of combined liver and kidney transplants in small children $(<15 \mathrm{~kg})$. Transplantation 88:711-715

12. Tait BD, Susal C, Gebel HM, Nickerson PW, Zachary AA, Claas FH, Reed E, Bray RA, Canpbell P, Chapman JR, Coates PT, Colvin RB, Cozzi E, Doxiadis II, Fuggle SV, Gill J, Glotz D, Lachmann N, Mohanakumar T, Suciu-Foca N, SumitranHolgersson S, Tanabe K, Taylor CJ, Tyan DB, Webster A, Zeevi 
A, Opelz G (2013) Consensus guidelines on the testing and clinical management issues associated with HLA and non-HLA antibodies in transplantation. Transplantation 95:19-47

13. Olausson M, Mjörnstedt L, Norder G, Rydberg L, Mölne J, Bäckman L, Friman S (2007) Successful combined partial auxiliary liver and kidney transplantation in highly sensitized crossmatch positive recipients. Am J Transplant 7:130-136

14. Dar W, Agarwal A, Watkins C, Gebel HM, Bray RA, Kokko KE, Pearson TC, Knechtle SJ (2011) Donor-directed MCH class I antibody is preferentially cleared from sensitized recipients of combined liver/kidney transplants. Am J Transplant 11:841-847

15. Creput C, Durrbach A, Samuel D, Eschewege P, Amor M, Kriaa F, Kreis H, Benoit G, Bismuth H, Charpentier B (2003) Incidence of renal and liver rejection and patient survival rate following combined liver and kidney transplantation. Am J Transplant 3:348-356

16. Fong TE, Bunnapradist S, Jordan SC, Selby RR, Cho YW (2003) Analyses of the United Network for Organ Sharing database comparing renal allografts and patient survival in combined liverkidney transplantation with the contralateral allografts in kidney alone or kidney-pancreas transplantation. Transplantation 76:348353

17. de la Cerda F, Jiminez WA, Gjertson DW, Venick R, Tsai E, Ettenger R (2010) Renal graft outcome after combined liver and kidney transplantation in children: UCLA and UNOS experience. Pediatr Transplant 14:459-464

18. Rogers J, Bueno J, Shapiro R, Scantlebury V, Mazariegos G, Fung J, Reyes J (2001) Results of simultaneous and sequential pediatric liver and kidney transplantation. Transplantation 72:1666-1670

19. Simpson N, Cho YW, Cicciarelli JC, Selby RR, Fong TE (2006) Comparison of renal allograft outcomes in combined liver-kidney transplantation versus subsequent kidney transplantation in liver transplant recipients: analysis of UNOS database. Transplantation 82:1298-1304

20. Creput C, Le Friec G, Bahari R, Amiot L, Charpentier B, Carosella E, Rouas-Freiss N, Durrbach A (2003) Detection of HLA-G in serum and graft biopsy associated with fewer acute rejections following combined liver-kidney transplantation: possible implications for monitoring patients. Hum Immunol 64:1033-1038

21. Ingelsten M, Karlsson-Parra A, Granqvist A, Mölne J, Olausson M, Haraldsson B, Nyström J (2011) Postischemic inflammatory response in an auxiliary liver graft predicts renal graft outcome in sensitized patients. Transplantation 91:888-894

22. Fargue S, Harambat J, Gagnadoux MF, Tsimaratos M, Janssen F, Llanas B, Berthélémé JP, Boudailliez B, Champion G, Guyot C, Macher MA, Nivet H, Ranchin B, Salomon R, Taque S, Rolland MO, Cochat P (2009) Effect of conservative treatment on the renal outcome of children with primary hyperoxaluria type 1. Kidney Int 76:767-773

23. Harambat J, vanStralen KJ, Espinosa L, Groothoff JW, Hulton SA, Cerkauskiene R, Schaefer F, Verrina E, Jager KT, Cochat P (2012) Characteristics and outcomes of children with primary oxalosis requiring renal replacement therapy. Clin J Am Soc Nephrol 7:458-465

24. Cochat P, Hulton SA, Acquaviva C, Danpure CJ, Daudon M, de Marchi M, Fargue S, Groothoff J, Harambat J, Hoppe B, Jamieson NV, Kemper MJ, Mandrile G, Marangella M, Picca S, Rumsby G, Salido E, Straub M, van Woerden CS, OxalEurope (2012) Primary hyperoxaluria type 1: Indications for screening and guidance for diagnosis and treatment. Nephrol Dial Transplant 27:1729-1736

25. Jamieson NV, The European PH1 Transplantation Study Group (2005) A 20-year experience of combined liver/kidney transplantation for primary hyperoxaluria (PH1): the European PH1 trasnsplant registry experience 1984-2004. Am J Nephrol 25:282-289

26. Brinkert F, Ganschow R, Helmke K, Harps E, Fischer L, Nashan B, Hoppe B, Kulke S, Muller-Wifel DE, Kemper MJ (2009) Transplantation procedures in children with primary hyperoxaluria type 1: outcome and longitudinal growth. Transplantation 87:1415-1421

27. Bergstrahl EJ, Monico CG, Lieske JC, Herges RM, Langman CB, Hoppe B, Millner DS, the IPHR investigators (2010) Transplantation outcomes in primary hyperoxaluria. Am J Transplant 10:2494-2501

28. Hoppe B (2012) An update on primary hyperoxaluria. Nat Rev Nephrol 8:467-475

29. Perera MT, McKiernan PJ, Sharif K, Milford DV, LLoyd C, Mayer DA, Kelly DA, Mirza DF (2009) Renal recovery in children undergoing combined liver kidney transplants. Transplantation $87: 1584-1589$

30. Scheinman JI, Alexander M, Campbell ED, Chan JC, Latta K, Cochat P (1995) Transplantation for primary hyperoxaluria in the USA. Nephrol Dial Transplant 10:42-46

31. Galanti M, Contreras A (2010) Excellent renal function and reversal of nephrocalcinosis 8 years after isolated liver transplantation in an infant with primary hyperoxaluria type 1 . Pediatr Transplant 25:2359-2362

32. Jalanko H, Peltonen S, Koskinen A, Puntila J, Isoniemi H, Holmberg C, Pinomäki A, Armstrong E, Koivusalo A, Tukiainen E, Mäkisalo H, Saland J, Remuzzi G, de Cordoba S, Lassila R, Meri S, Jokiranta TS (2008) Successful liver-kidney transplantation in two children with aHUS caused by a mutation in complement factor $\mathrm{H}$. Am J Transplant 8:216-221

33. Saland JM, Emre SH, Schneider BL, Benchimol C, Ames S, Bromberg JS, Remuzzi G, Strain L, Goodship TH (2006) Favorable outcome after liver-kidney transplant for recurrent hemolytic uremic syndrome associated with factor $\mathrm{H}$ mutation. Am J Transplant 6:1948-1952

34. Loirat C, Saland J, Bitzan M (2012) Management of hemolytic uremic syndrome. Presse Med 41:e115-e135

35. Saland JM, Ruggenenti P, Remuzzi G, Consensus Study Group (2009) Liver-kidney transplantation to cure atypical hemolytic uremic syndrome. J Am Soc Nephrol 20:940-949

36. Zuber J, Le Quintrec M, Krid S, Bertoye C, Gueutin V, Lahoche A, Heyne N, Ardissino G, Chatelet V, Noel LH, Hourmant M, Niaudet P, Frémeaux-Bacchi V, Rondeau E, Legendre C, Loirat C, French Study Group for Atypical HUS (2012) Eculizumab for atypical hemolytic uremic syndrome recurrence in renal transplantation. Am J Transplant 12:3337-3354

37. Kasahara M, Horikawa R, Tagawa M, Uemoto S, Yokoyama S, Shibata Y, Kawano T, Kuroda T, Honna T, Tanaka K, Saeki M (2006) Current role of liver transplantation for methylmalonic academia: a review of the literature. Pediatr Transplant 10:943-947

38. McGuire PJ, Lim-Melia E, Diaz GA, Raymond K, Larkin A, Wasserstein MP, Sansaricq (2008) Combined liver-kidney transplant for the management of methylmalonic aciduria: a case report and review of the literature. Mol Genet Metab 93:22-29

39. Chen PW, Hwu WL, Ho MC, Lee NC, Chien YH, Ni YH, Lee PH (2010) Stabilization of blood methylmalonic acid level in methylmalonic acidemia after liver transplantation. Pediatr Transplant 14:337-341

40. Lubrano E, Elli M, Rossi M, Travasso E, Raggi C, Barsotti P, Carducci C, Berloco P (2007) Renal transplant in methylmalonic academia: could it be the best option? Report on a case at 10 years and review of the literature. Pediatr Nephrol 22:1209-1214

41. Kaplan P, Ficicioglu C, Mazur AT, Palmeri MJ, Berry GT (2006) Liver transplantation is not curative for methylmalonic acidopathy caused by methylmalonyl-CoA mutase deficiency. Mol Genet Metab 88:322-326

42. Clothier JC, Chakrapani A, Preece M-A, McKiernan P, Gupta R, Macdonald A, Hulton S-A (2011) Renal transplantation in a boy with metylmalonic acidemia. J Inherit Metab Dis 34:695-700

43. Loreno M, Boccagni P, Rigotti P, Naccarato R, Burra P (2002) Combined liver-kidney transplantation in a 15 -year-old boy with alpha-1-antitrypsin deficiency. J Hepatol 36:565-568 
44. Panaro F, Andorno E, Basile G, Morelli N, Bottino G, Fontana I, Bertocchi M, DiDomenico S, Miggino M, Saltalamacchia L, Ghinolfi D, Bonifazio L, Jarzemboeski TM, Valente U (2004) Simultaneous liver-kidney transplantation for glycogen storage disease type Ia (von Gierke's disease). Transplant Proc 36:14831484

45. Marega A, Fregonese C, Tulissi P, Vallone C, Gropuzzo M, Toniutto PL, Baccarani U, Bresadola F, Toso F, Montanero D (2011) Preemptive liver-kidney transplantation in von Gierke disease: a case report. Transplant Proc 43:1196-1197

46. Srinath A, Schneider BL (2012) Congenital hepatic fibrosis and autosomal recessive polycystic kidney disease. JPGN 54:580587

47. Khan K, Schwarzenberg SJ, Sharp HL, Matas AJ, Chavers BM (2002) Morbidity from congenital hepatic fibrosis after renal transplantation for autosomal recessive polycystic kidney disease. Am J Transplant 2:360-365

48. Davis ID, Ho M, Hupertz V, Avner ED (2003) Survival of childhood polycystic kidney disease following renal transplantation: the impact of advanced hepatobiliary disease. Pediatr Transplant 7:364-369

49. D'Amico G, Pagliaro L, Bosch J (1995) The treatment of portal hypertension: a meta-analytic review. Hepatology 22:332-356
50. Chapal M, Debout A, Dufay A, Salomon R, Roussey G, Burtey S, Launay EA, Vigneau C, Blancho G, Loirat C, Hourmant M, Fakhouri F (2012) Kidney and liver transplantation in patients with autosomal recessive polycystic kidney disease: a multicentric study. Nephrol Dial Transplant 27:2083-2088

51. Davis CL, Feng S, Sung R, Wong F, Goodrich NP, Melton LB, Reddy KR, Guidinger MK, Wilkinson A, Lake J (2007) Simultaneous liver-kidney transplantation: evaluation to decision making. Am J Transplant 7:1702-1709

52. Fong TE, Khemichian S, Shah T, Hutchinson IV, Cho YW (2012) Combined liver-kidney transplantation is preferable to liver transplant alone for cirrhotic patients with renal failure. Transplantation 94:411-416

\section{Answers}

1. a

2. b

3. c

4. c

5. b 